\title{
A High-Speed Intelligence Smart Grid System by Using MIMO Channel Capacity
}

Prakash Thapa, Shree Krishna Acharya, Hui Il Chang, Suji Park and Hyun Su Jung, Prof. Oh Il Whan, Gye Choon Park and Jin Lee

\begin{abstract}
Smart grid system is the upcoming power control system working as autonomous system by incorporating number of energy resources, two way wire and wireless communication. A reliable operation requires multiple, high-data-rate, two-way communications links among all communicative nodes of smart grid network, which do not exist today, plus powerful computing facilities at the control center. A control signal is generated by number of sensor in real time which is provide to micro computer system and Advanced Metering Infrastructure (AMI). Disturbance tracking, communicative on each node and fault awareness is major facing issues of Smart grid which is still in extinct. Large geographic area of earth surfaces is the main reason for getting such problems. In this paper, we used broadcasting by multi-user MIMO system on smart grid network that provides high speed two way communication over large geographical area by adopting cellular network. Such type of Smart grid is capable for accepting real time active user and provide two way autonomous system with high data capacity rate. Each real -time active user with efficient data bit-stream probability and active sensor control signals, MIMO system easily enhance the communication capability of smart grid network.
\end{abstract}

Keywords-Smart Grid System, AMI, MIMO Channel Capacity

\section{Introduction}

The smart grid is considered with new features and capabilities such as high penetration of renewable energy source (RES), two -way energy demanding and demand-side management (DSM) which clearly holds exploiting the potential of future in wireless communication. Toady's challenge of smart grid system is track disturbance in real time which means power grid should be responsible, awake and communicative on every node. Smart grid utilizes concept of efficient spectrum and Advanced Metering Infrastructure system (AMI). The demands of smart grid system is increasing day by day due to efficient real time working through wire and wireless. Energy management system (EMS) is computer control in smart grid which is more developed in rich countries. Such system provide optimum price affordable quality of power supply to local consumers.
A micro computer system that sort the automated power network which is highly fabricated with centralized control systems known as Supervisory Control and Data Acquisition (SCADA) systems. With the help of SCADA an intelligent distributed micro grids [1-2] is developed with sensitive and advanced measurement functions. Toady's challenge of smart grid system is track disturbance in real time which means power grid should be responsive, awake and communicative with every node.

A cellular communication system is quick and inexpensive to obtain communication coverage in large geographic area so many researcher conducted research such as back-to end nodes using wide area network communication with sensor/relay nodes. [3]. The cost factor and broadband communication performance are main reason of integrated smart grid system which promising wired and wireless technology such as Wi-Fi, worldwide interoperability for microwave access (WiMAX), Ethernet, General packet radio services (GPRS), ZigBee, HART, WLAN 802.11, RF Mesh, and multicarrier wireless information local loop (McMill). [4]

A practical smart grid models which coordinated with cellular networks are developed [5-9] that work on energy efficient resource allocation, energy management, MIMO -downlink transmission, and transmit-beam forming design for minimize the cost factor. However, none of these works addressed on rate limits and signal to noise ratio (SNR) for individual user -broadcasting channel. LTE and $4 \mathrm{G}$ are new generation wireless communication technologies provides wider area of application as their characteristics of bidirectional communications and wider network which is more suitable for wide spreading and remotely control. [10].

In this paper, we introduce broadcasting in multiuser MIMO system with its SNR capability. Base station are working as a Gateways. It provides efficient data speed to the active user. Gateways are always active for accepting response. Multi-user phenomena of MIMO provides a reliable data rate to each active user with efficient data bit-stream probability. Receiving wireless bit-stream is depend upon number of real -time active user of smart grid system. Wireless bit stream is the combination of 
active sensor control signals which is autonomously controlled by each active user.

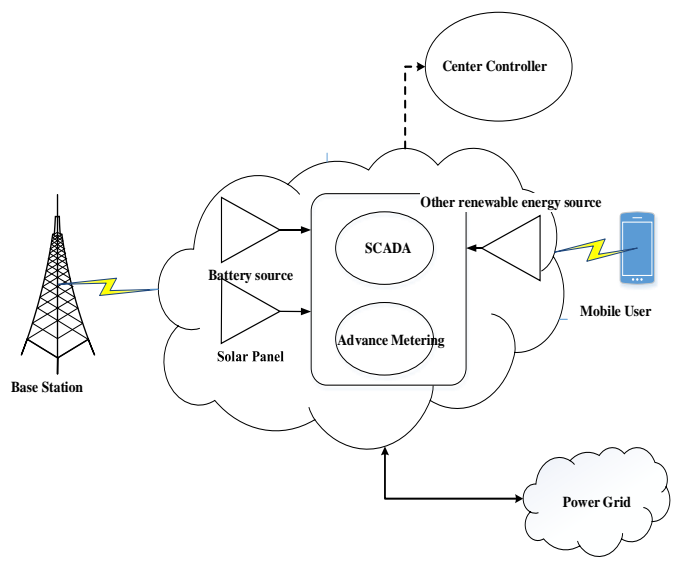

Figure 1 Integrated autonomous smart grid system

User can be moved in large geographic area. Base station is responsible to communicate with metering system through wirelessly. Each active user is defined in real time basis. A high speed control signal is transmitted to active user.

\section{Intelligence Smart Grid System}

The high demand of computer based control systems leads to strategic control and management planning. Conventional power grid system has four separate elements that are generations plants, transmission, distribution networks, and end users.[11] As they are separate elements, they can do function together only when smart system has effective data communication networking systems [12]. However, we can minimize a cost factor by controlling every segment separately by integrated computer communication.

The demands of smart grid system is increasing day by day due to efficient real time working through wire and wireless. However, its working is limited in some issues like working area, power management etc. To remove this effect our designing system prefers to wireless autonomous control of smart analysis.

An intelligence smart grid (SG) system autonomously collect a real-time data, analysis it by using information about cyber security, computational intelligence, electricity generation, substations, distribution and consumer consumption, and provide secure, safe and reliable control. [13]

The data information from various terminal which is electrically interconnected utility domain handle by SCADA system. Electrical information network protocol [EINP] is a part of SCADA that help in monitoring, controlling, configuration and troubleshooting the electric power networks.

\section{AMI -System}

Advanced AMI system is intelligence based system which calculate all power of the system in real time and aware to communicate with Gateways. Capability of maintaining balanced power system in the smart grid connection simply overcome black-out effect. Modern AMI system is responsible for consumer involvement and their load management, industrial load management, national energy management, economic security management. The demands of versatile AMI infrastructure accepting friendlier management to mobile user due to cellular communication system. The communication node implemented with MIMO system which is able to scan and identify some available channels which simply ignore the inactive users for a unit time to maintain high speed data communication.

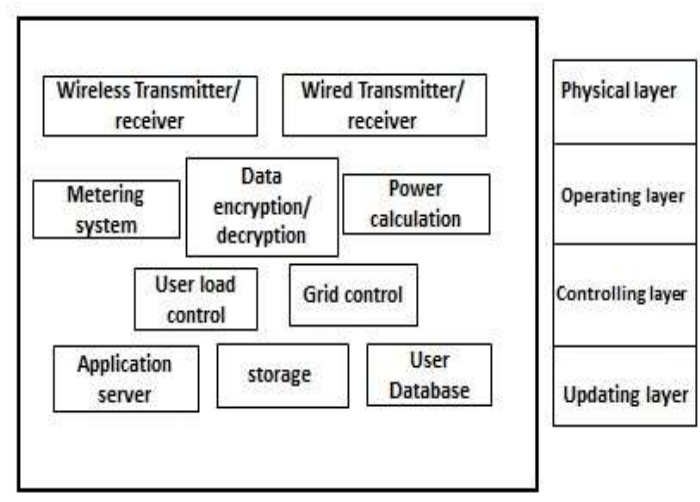

Figure 2 Smart Metering system of end user

A digital network based AMI-meter is replace the decades-old power meter, which works in gear turning, with the help of layer wise processing that track not only out-going and out-going current but also communicate with end-user through wireless and wired communication. An AMI is capable to communication with end user using fourth generation MIMO technology.

\section{Iv. Wireless Communication Control Management}

Wireless networks are anticipated to be more dominant in Smart grid (SG) is especially wireless mesh networks (WMNs) due to reliable and ability 
of providing redundant communications paths. [13] Cost effective sensing and communication system control and diagnosis attract many researcher for implementation on smart grid by wireless communication channel by using WSNs. [14-16] Operation related to power system contingency detection and isolation to avoid cascade effects may leads to failure but we have some promising wireless and wired technology in smart grid system. Wireless device fundamentally works on sensing and communication control mechanism such as wireless sensor networks (WSNs), wireless regional area network (WRAN), power sensor, and wireless router as shown in figure.

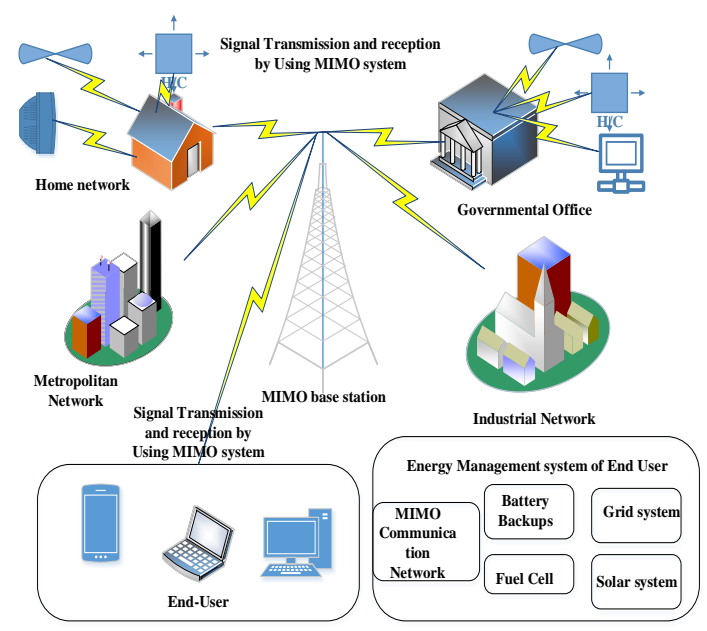

Figure 3 Large geographical coverage high speed smart grid network using MIMO configuration

\section{v. MIMO Channel Capacity}

The MIMO system increases the channel capacity as number of transmitting antenna and receiving antennas are increases. The effect of statically dependence of discrete transmitting and noise vector signal provide a useful improvement of the channel capacity of the MIMO system. The measuring distance between two probability distribution which gives amount of information of one to another random variables which is also known as Mutual information. Mutual information is mainly depends upon the varying PDF of transmit signal vector. The definition of Entropy is becomes same with mutual information when channels input and output are vector values instead of scalar.

The channel capacity is the maximum Mutual Information obtain from transmit signal vector by varying its pdf. We can express in terms of entropy as

$$
I(x ; y)=H(y)-H(y \mid x) .
$$

where $H(y \mid x)=H(z)$ is the noise signal when noise signal $\mathrm{X}$ and $\mathrm{Y}$ are statically independence. The maximum capacity can be calculated when all signal are in ZMSCG. For single user MIMO system channel capacity becomes,

$$
\mathbf{I}(\mathbf{X} ; \mathbf{Y})=\log _{2}\left(\operatorname{det}\left(\pi e\left(R_{y y}\right)\right)+\log _{2}\left(\operatorname{det}\left(\pi e\left(\pi e N_{0} I_{N_{n}}\right)\right) \ldots(2)\right.\right.
$$

Channel state information are known to the receiver, then our capacity of MIMO system will be

$$
C=\underset{T r(R x x)=N T}{\operatorname{Max}} \log _{2}\left(I_{N_{A}}+\frac{E x}{N_{T} N_{0}} * H R_{X X} H^{H}\right)
$$

\section{Multi-User MIMO Model of Smart Grid System}

Our system uses multi-user MIMO system for wireless communication. Multi-user MIMO working on the uplink and downlink channel. Spatial multiplexing is the key feature of the MIMO system which provide point to point high data rate in Single -user MIMO system with spatial gain. However, today's communication system deals with multi-user communication environment where multiple user are handled by same base station also known as gateways on smart grid terms.

If we have $\mathrm{K}$ user in our gateways which communicate virtual set of antenna which are equipped with $N_{B} \& N_{M}$.For the end to end communication, we considered an active user within that gateways so MIMO system for downlink will be $\left(K_{\text {act }} \cdot N_{M}\right) \times N_{B}$ and MIMO system for uplink will be $N_{B} \times\left(K \cdot N_{M}\right)$ on smart grid system. Facility to the number of user in smart grid system provided by uplink channel in smart grid. Let we have $u=1,2, \ldots \ldots K_{\text {act }}$ active end user which produce $X_{u} \in \mathbb{C}^{N_{M} \times 1}$ received signal at the Base Station and corresponding transmit signal is $Y \in \mathbb{C}^{N_{B} \times 1}$ then the channel gain between $u^{\text {th }}$ active user and gateways is represented by $H_{u}^{U P} \in \mathbb{C}^{N_{B} \times N_{M}}$ and given by

$$
Y=\sum_{u=0}^{k} H_{K_{\text {act }}}^{U P} \times X_{K_{\text {act }}}+Z
$$

where $Z \in \mathbb{C}^{N_{B} \times 1}$ is the additive Zero-mean circular symmetric complex Gaussian (ZMCSCG) random vector. Linear Pre-equalization method uses Channel State Information (CSI) for Spatial multiplexing system. A number of symbol bits are transmitted from end-users received by Gateways. A pre-equalizer weight matrix $W \in \mathrm{C}^{N_{T} \times N_{R}}$ and the pre-coded symbol vector is $X_{k}=C^{N_{T} \times 1}=W \check{x}$ which included weight form as.

$$
X_{k}=W \tilde{x}_{m}
$$


In MIMO system pre equalization scheme on the transmitter side outperforms then a receiver side. The weight vector can be

$$
W=\beta \times H^{H}\left(H H^{H}+\frac{\sigma_{z}^{2}}{\sigma^{2}{ }_{x}} * I\right)^{-1}
$$

where $\beta$ is total power transmitted constraint.

$$
\beta=\sqrt{\frac{N_{T}}{\operatorname{Tr}\left(H^{-1}\left(\left(H^{-1}\right)^{H}\right)\right)}} \ldots \ldots \ldots \ldots \ldots \ldots \ldots \ldots(7)
$$

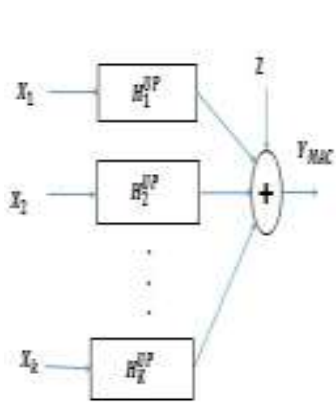

|a)

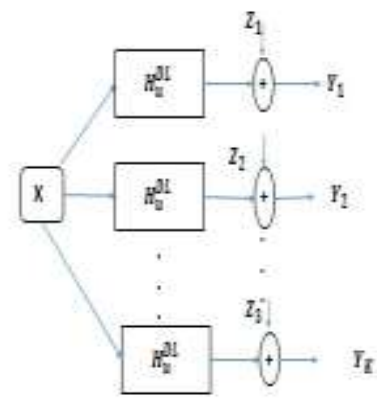

(2)

Figure 4 multi-user MIMO system (a) uplink Model and (b) downlink Model

The co-ordinate selection in downlink is not straight forward because of interference cancellation. The resolving of such problem is known as Channel Inversion where received signals of all users can be found in scalar form. Each user on smart grid system treat with high data rate.

\section{Simulation}

The capacity of MIMO system depend upon the number of transmitting antenna and receiving antenna. End user can get reliable two-way communication path. For simulation we take a single user MIMO to determine the capacity rate on various antenna system. The below figure 5 shows user data rate capability.

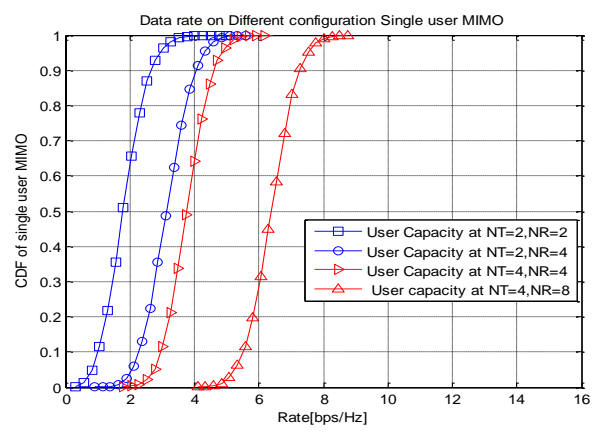

Figure 5 Channel capacity of single user MIMO
For finding number of active and passive end user with corresponding receiving symbol probability we use QPSK mapping in Multi-user MIMO system. Figure 6 shows symbol bit probability on individual user in smart grid systems. A QPSK mapper and demapper is used for receiving high probability signal in active user side. Smart Grid with various MIMO configuration which provide high probability signal to the active user is shown below.

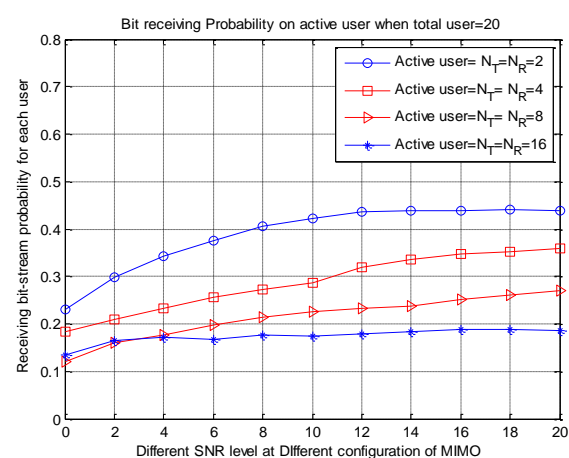

Figure 6 Active user bit accessing probability of smart grid system control by MIMO

Signal to Noise ratio (SNR) is very important for smart metering system to detect end user command for doing operation. It is found that a MIMO system has good channel capacity when its SNR is increases.

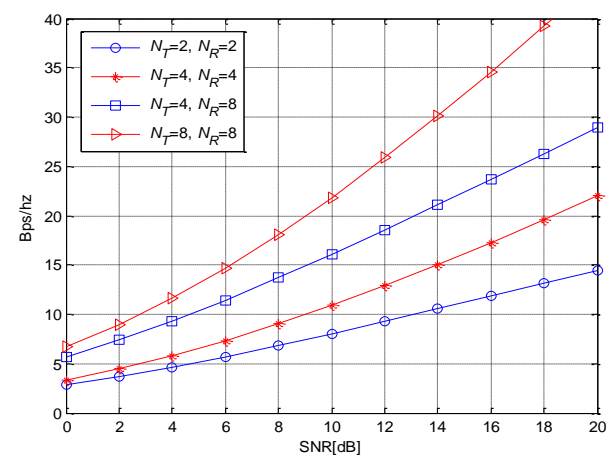

Figure 7 SNR analysis of single user MIMO

\section{viII. Conclusion}

A smart grid system by using MIMO concept enhanced the closed loop system within large geographical area maintaining a high data rate transmission. Selection of active user in real time effectively provides the space for active control sensor signals. An intelligence smart grid system consists of large number of sensor control signal which is acceptable by the MIMO configuration. High data rate containing number of sensor control data and capability of selecting active user of respective gateways estimate an intelligence smart grid system. 


\section{Acknowledgment}

This paper is prepared under Smart Energy Campus project-2016 supported by KEPCO.

\section{References}

[1] D. J. Cox, J. Tom, Distributed Generation and Sensing for Intelligent Distributed Micro grids, Proceedings of the 2006 IEEE/SMC International Conference on System of Systems Engineering Los Angeles, CA, USA - April 2006

[2] Y. Li, D. M. Vilathagamuwa, and P. C. Loh, "Design analysis and real time testing of a controller for multibus microgrid system," IEEE transactions on Power Electronics, 19 (2004) pp. 1195-1204,Sept 2004.

[3] B.Akyol, H. Kirkham, S. Clements and M. Hadley, "A survey of wireless communications for the electric power system", Prepared for the U.S. Department of Energy, (2010).

[4] S. Ullo, A. Vaccaro, G. Velotto, The role of pervasive and cooperative sensor networks in Smart Grids communication, in: MELECON 2010-2010 15th IEEE Mediterranean Electrotechnical Conference, 2010, pp. 443-447.

[5] S. Bu, F. Yu, Y. Cai, and X. Liu, "When the smart grid meets energyefficient communications: Green wireless cellular networks powered by the smart grid," IEEE Trans. Wireless Commun., vol. 11, no. 8, pp.3014-3024, Aug. 2012

[6] J. Xu, Y. Guo, and R. Zhang, "CoMP meets energy harvesting: A new communication and energy cooperation paradigm," IEEE Trans. Veh. Technol., vol. 64, no. 6, pp. 2476-2488, Jun. 2015.

[7] Dynamic Resource Allocation for Smart-Grid Powered MIMO Downlink Transmissions X Wang, T Chen, X Chen, X Zhou, GB Giannakis - 2016

[8] X. Wang, Y. Zhang, G. B. Giannakis, and S. Hu, "Robust smartgrid powered cooperative multipoint systems," IEEE Trans. Wireless Commun., vol. 14, no. 11, pp. 6188-6199, Nov. 2015

[9] X. Wang, Y. Zhang, T. Chen, and G. B. Giannakis, "Dynamic energy management for smart-grid powered coordinated multipoint systems," IEEE J. Sel. Areas Commun., to appear 2016.

[10] Zhao Feng; Zhang Yuexia; , "Study on smart grid communications system based on new generation wireless technology," Electronics, Communications and Control (ICECC), 2011 International Conference on , vol., no., pp.1673-1678, 9-11 Sept. 2011.

[11] NIST, NIST framework and roadmap for Smart Grid interoperability standards, Release 1.0, January 2010. Accessed on 1st November, 2012.

[12] V.K. Sood, D. Fischer, J.M. Eklund, T. Brown, Developing a communication infrastructure for the Smart Grid, in: Electrical Power \& Energy Conference, EPEC, 2009 IEEE, 2009, pp. 1-7.

[13] Fang, X., Misra, S., Xue, G., Yang, D, Smart Grid - The New and Improved Power Grid: A Survey, Communications Surveys \& Tutorials, IEEE, 14 (2011), 944-980.

[14] N. Bressan, L. Bazzaco, N. Bui, P. Casari, L. Vangelista, and M. Zorzi. The deployment of a smart monitoring system using wireless sensors and actuators networks. IEEE Smart Grid Comm'10, pages 49-54, 2010.

[15] B. Lu, T. G. Habetler, R. G. Harley, and J. A. Guti'errez. Applying wireless sensor networks in industrial plant energy management systems-part I: A closed-loop scheme. IEEE Sensors, pages 145-150, 2005.
[16] B. Lu, T. G. Habetler, R. G. Harley, J. A. Guti'errez, and D B. Durocher. Energy evaluation goes wireless. IEEE Industrial Applications Magazine, 13(2007), 17-23.

[17] L. K. Siow, P. L. So, H. B. Gooi, F. L. Luo, C. J. Gajanayake, Q. N. Vo., Wi-Fi Based Server in Microgrid Energy Management System. TENCON 2009-IEEE Region 10 Conference.

[18] Smart Grids European Technology Platform. SmartGridsStrategic Deployment Document for European Electricity Networks of the Future. Available online: (accessed on 7 January 2012)

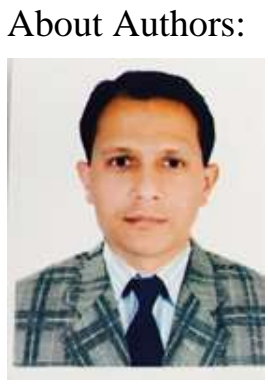

Prakash Thapa

Completed Bachelor degree in 2008 from TU, Nepal and served 6years in renewable energy sector of Nepal as an Engineer. Currently doing Master degree in Mokpo National University, South korea, and interested in Wireless Sensor and Networking, Fuel Cell, Smart Grid Energy System, Cognitive Radio, MIMO etc.

\section{Other Authors are:}

Name: Shree Krishna Acharya (Author) University: Mokpo National University, Country: South korea.

Name: Hui Il Chang (Corresponding Author) University: Gwgangju University, Country: South korea.

Name: Prof. Il Whan Oh

University: Mokpo National University Country: South Korea

Name: Gye Choon Park

University: Mokpo National University, Country: South korea.

Name: Jin Lee

University: Mokpo National University, Country: South korea.

Name: Suji Park

University: Mokpo National University, Country: South korea.

Name: Hyun Su Jung

University: Mokpo National University, Country: South korea. 